\title{
Reflective meniscometry: a non-invasive method to measure tear meniscus curvature
}

\author{
Norihiko Yokoi, Anthony Bron, John Tiffany, Nicholas Brown, James Hsuan, Colin Fowler
}

Department of Ophthalmology, Kyoto Prefectural University of Medicine, Kyoto, Japan

N Yokoi

Nuffield Laboratory of Ophthalmology,

University of Oxford, Oxford

A Bron

J Tiffany

N Brown

J Hsuan

Department of Physiological Optics, University of Aston, Birmingham

C Fowler

Correspondence to: Anthony J Bron, Nuffield Laboratory of Ophthalmology,

University of Oxford, Walton Street, Oxford OX2 6AW.

Accepted for publication 29 July 1998

\begin{abstract}
Aims-To devise a method to measure tear meniscus curvature by a non-invasive specular technique.

Methods-A photographic system was devised. The system consisted of a camera and an illuminated target with a series of black and white stripes oriented parallel to the axis of the lower tear meniscus. The target was mounted on a flash gun close to the objective of a Brown macrocamera and calibrated using a graduated series of glass capillaries of known diameter, ground down to expose the inner wall. It was then applied to normal human eyes $(n=45)$ to measure the tear meniscus curvature. A video system was also assessed which provided qualitative online information about the tear meniscus.

Results-Using the photographic system, measured values for capillary radii were in excellent agreement with theoretical calculations $\quad\left(r^{2}=0.996, p<0.0001\right)$. The radii of curvature of lower tear menisci in normal human subjects (mean 0.365 (SD $0.153) \mathrm{mm}$, range $0.128-0.736 ; n=45)$ were similar to those reported in the literature. Both systems demonstrated variations in meniscus shape. The video system provided stable images of human menisci over prolonged periods of time and promises to be useful for the analysis of dynamic changes in meniscus volume. Conclusions-Reflective meniscometry is a non-invasive technique providing quantitative information about tear meniscus shape and volume and of potential value in the study of ocular surface disease.

(Br f Ophthalmol 1999;83:92-97)
\end{abstract}

Human tears are distributed between the culs de sac $(4.5 \mu \mathrm{l})$, menisci $(2.9 \mu \mathrm{l})$, and preocular tear film $(1.1 \mu \mathrm{l}) .{ }^{1}$ Of these, the meniscus provides a reservoir which accommodates excess tears during reflex tearing, ${ }^{2}$ in dysfunction of the lacrimal drainage system, ${ }^{3}$ or after drop instillation. In the tear deficient dry eye, the meniscus volume is known to fall ${ }^{4}$ with the fall in lacrimal secretory rate. ${ }^{5}$

In the diagnosis of ocular surface diseases, including dry eye, information about tear volume and rate of flow is indispensable, ${ }^{67}$ and currently the Schirmer and cotton thread tests $^{89}$ are widely established methods of measurement. Alternatively, meniscus variables such as height, width, radius of curvature, and cross sectional area are also reported to be of value in the diagnosis of dry eye. ${ }^{40-16}$ However, all of the above techniques are limited by their invasive nature ${ }^{17} 18$ (involving the insertion of papers, threads, or fluorescein into the tear film), which either add fluid to the conjunctival sac or can stimulate tearing.

We report here a novel, non-invasive specular method for measuring tear meniscus curvature, which uses the tear strip as a concave mirror. We term this technique reflective meniscometry. Its application to the human tear meniscus is described and the values are compared with reported values. ${ }^{16}{ }^{19}$ The method is expected to have a wide field of application to the analysis of ocular surface disease.

\section{Methods}

PHOTOGRAPHIC SYSTEM

The system is illustrated in Figures 1 and 2, and consists of a camera, target and flash source. A Brown macrocamera (Marcher, Hereford) is used..$^{20}$ The target consists of a set of horizontal black and white stripes (each 2 mm wide; black 14; white 13) printed onto acetate sheet and mounted on a flash gun (Jessop $100 \mathrm{M}$, Leicester) attached by adjustable metal arms to the lens tube of the camera. The target was constructed using MacDraw Pro (Claris Corp, USA) on a Macintosh PowerBook 5300cs personal computer. A diffusing screen was interposed between flash and target, and the side closer to the camera was independently illuminated by two miniature filament lamps (12 V, 60 mA; RS Components, Corby). For photography, the camera and target were each set at about 20 degrees to the sagittal plane, with the subject's head resting comfortably at the slit lamp. The target was set at about $27 \mathrm{~mm}$ from the meniscus, the exact distance from the lid margin being recorded with calipers. The film used was Kodak Elite II (ISO 100) and the usual lens aperture was $\mathrm{f} / 5.6$. The magnification on film was $\times 10$.

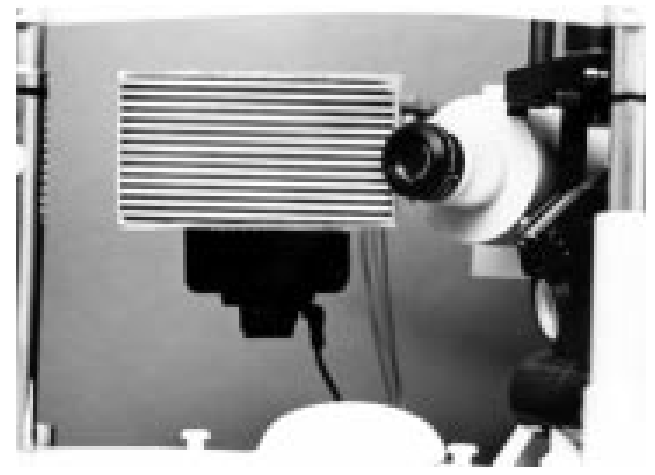

Figure 1 Front view of the photographic system, consisting of the target and a Brown macrocamera. 


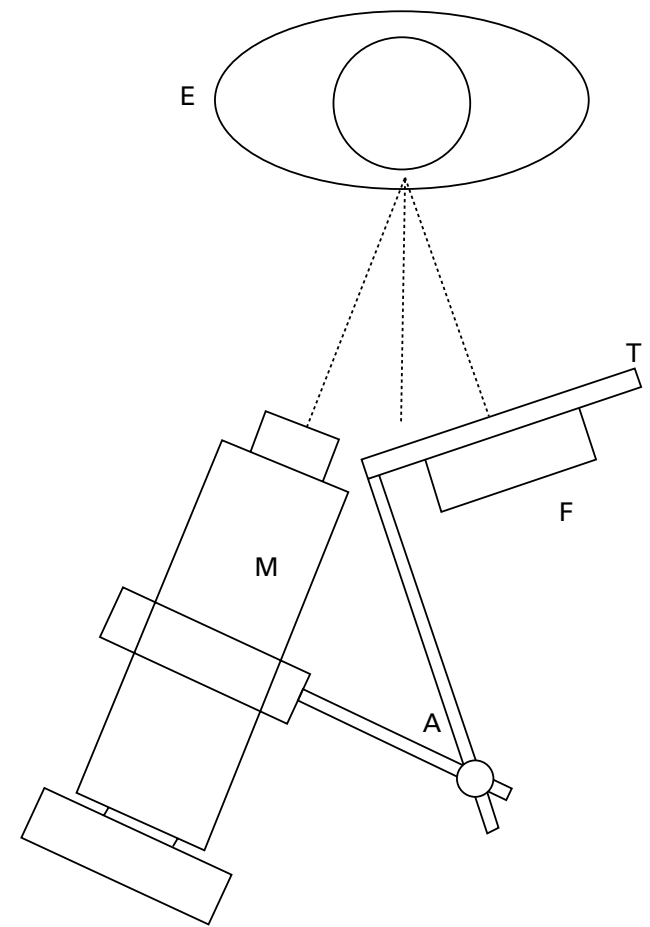

Figure 2 Diagram of the photographic system. E=subject's eye; $M=$ Brown's macrocamera; $T=$ target; $F=$ flash; $A=$ flexible arm.

VIDEO SYSTEM

A video system was also constructed to facilitate the study of qualitative changes in meniscus shape over time. A block diagram of the video system is shown in Figure 3. The system consists of a $50 \mathrm{~mm}$ objective lens (Schneider-Kreuznach, Germany) mounted on a bellows system permitting variation of magnification. This is linked to a COHU high performance, charge coupled device (CCD) video camera and TV monitor (Hitachi CPT2174, UK). The monitor is connected to a VHS video recorder (Sony SLV-270UB, Tokyo, Japan) for online recording. The target consists of a central white bar ( $3.5 \mathrm{~mm}$ wide) on a black surround, illuminated by a fibre optic source (Schott KL 150B, Mainz, Germany). Use of a single white bar facilitates on-screen measurement. Magnification can be calculated by imaging a standard graticule ( 40 lines/mm; Graticule Ltd, Kent), and in use the magnification on screen was in the region of $\times 60-70$.

The photographic system was calibrated using a series of disposable glass capillary tubes (5-50 $\mu \mathrm{l}$; Dade Diagnostics, Inc, USA; Supracaps; Blaubrand, Germany; Drummond Scientific, USA), whose outer halves were ground away using 1000 grit waterproof abrasive paper (silicon carbide) to expose the polished inner wall. These were mounted in front of the camera to act as concave reflecting surfaces of known curvature. The radius of curvature of each capillary was calculated to be 0.204 , $0.289,0.324,0.458$, and $0.547 \mathrm{~mm}$, based on the assumption of uniform cross sections along the length of the tubes. The average width of a single stripe in the image of the target in the vertical plane was calculated from the average

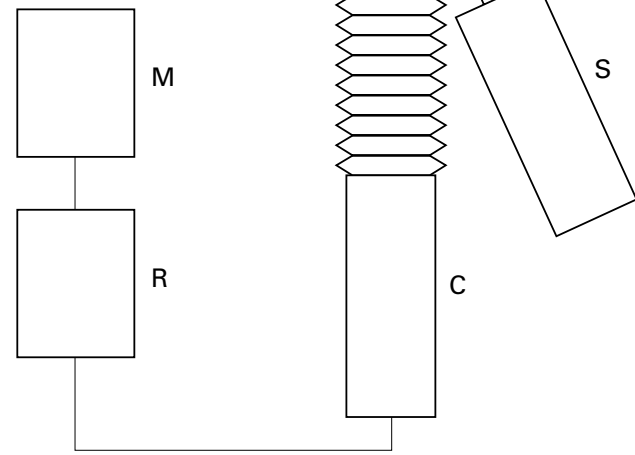

Figure 3 Diagram of the video system. E=subject's eye; $L=$ lens; $T=$ target; $S=$ light source; $C=$ video camera; $R=$ video recorder; $M=$ monitor.

width of three central cycles of black and white lines, enlarging with a Zeiss Dokumator (Carl Zeiss, Jena, Germany; magnification $\times 17.5$ ) and measuring with a millimetre rule. The central zone was selected to minimise problems of photographic parallax or noncircularity of the meniscus profile.

For the theoretical calculation of the radius of meniscus curvature, the equation for a concave mirror was used, ${ }^{21}$ in which the relation between image size and meniscus curvature is given by the formula: $r=2 /(\mathrm{L} / \mathrm{M}+\mathrm{L})$, where: $\mathrm{r}=$ radius of curvature of the meniscus, $\mathrm{L}=1$ / target distance, $M=$ magnification of the system. The derivation of this equation is given in the appendix.

APPLICATION OF REFLECTIVE MENISCOMETRY TO THE HUMAN TEAR MENISCUS

Studies with the photographic system included: (1) measurement of normal meniscus curvature, (2) measurement of curvature following a single flash, and (3) documentation of meniscus shape. Preliminary studies using the video system included: (a) observation of temporal changes in meniscus shape, (b) assessment of the effect of prolonged eye opening, and (c) assessment of the effects of drop instillation. This research conformed to the tenets of the Declaration of Helsinki and was approved by the Central Oxford Research Ethics Committee. Informed consent was obtained after a full explanation of the procedures.

A total of 45 normal volunteers were recruited (18 males; 27 females) who had no biomicroscopic abnormality of the cornea, conjunctiva or meibomian glands, no lacrimal drainage problems, no sign of dry eye and no history of contact lens wear. The mean age was 45.6 (SD 21.0) years. All measurements were made on the meniscus of the left eye, in the region of the centre of the lower lid. Repeatability was tested by taking two photographs in succession at an interval of 20 seconds, with the patient's head remaining at the slit lamp. In order to minimise any effect of gaze position on 

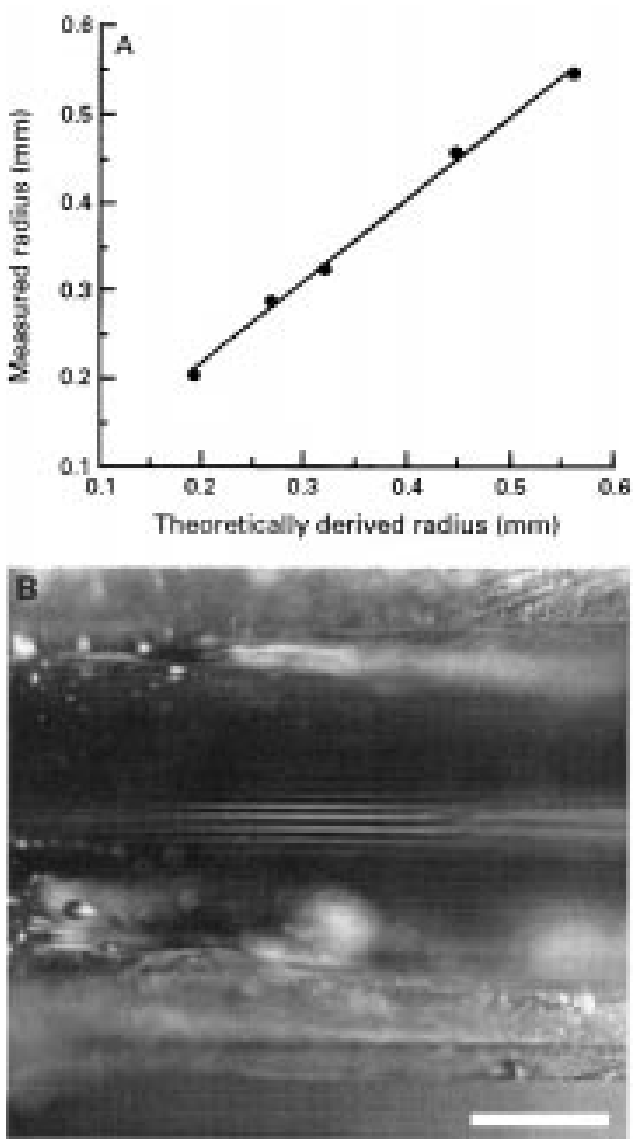

Figure 4 (A) Relation between measured and theoretically derived radius of curvature of an individual capillary tube target. Excellent agreement is shown: $\left(y=0.0300+0.936 x\left(r^{2}=0.996, p<0.0001\right)\right.$; where $x=$ theoretical ( $\mathrm{mm}), y=$ measured $(\mathrm{mm}))$. (B) Image formed by a ground down glass capillary tube (radius $0.547 \mathrm{~mm}$; bar $=500 \mu \mathrm{m})$.

meniscus shape, subjects were asked to look straight ahead in the horizontal plane and were encouraged to blink spontaneously. The humidity and temperature of the room as monitored daily were: mean temperature $20.5^{\circ} \mathrm{C}\left(\mathrm{SD} 1.5^{\circ} \mathrm{C}\right.$ ); mean humidity: $44.1 \%$ $(7.2 \%), \mathrm{n}=22$.

\section{Results}

CALIBRATION

Although from eight to 23 target lines (mean 15.5, SD 3.1) could be imaged on film, only the central three cycles of black and white stripes on any photograph (that is, six lines) were selected to obtain the average line width to minimise problems of parallax. A graph of this averaged vertical line width against radius of capillary curvature gave a straight line relation $\left(\mathrm{y}=0.039+2.54 \mathrm{x}\left(R^{2}=0.996, \mathrm{p}=\right.\right.$ $0.0014) ; \mathrm{x}=$ average line width $(\mathrm{mm})$ of the image in the vertical plane, $y=$ radius $(\mathrm{mm})$ of capillary curvature). Moreover, there was an excellent agreement between the theoretically derived and the measured radius of capillary curvature $\left(y=0.030+0.936 \mathrm{x} \quad\left(R^{2}=0.996\right.\right.$, $\mathrm{p}<0.0001) ; \mathrm{x}=$ theoretical $(\mathrm{mm}), \mathrm{y}=$ measured $(\mathrm{mm})$; Figure 4). These results indicate the precision of the photographic system for measurement of the meniscus curvature and incidentally confirm that the region selected

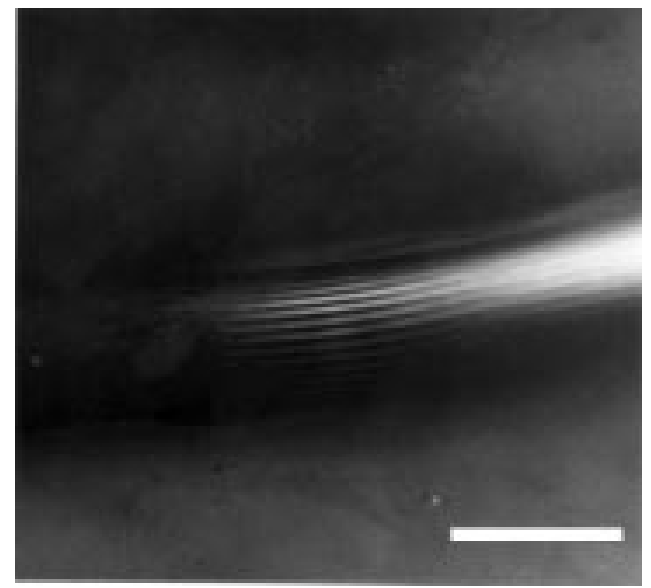

Figure 5 A representative example of the normal meniscus of a human eye. Measurement is made in the region of the central part of the lid where the images of the target lines are usually parallel (see text; bar $=500 \mu \mathrm{m}$ ).

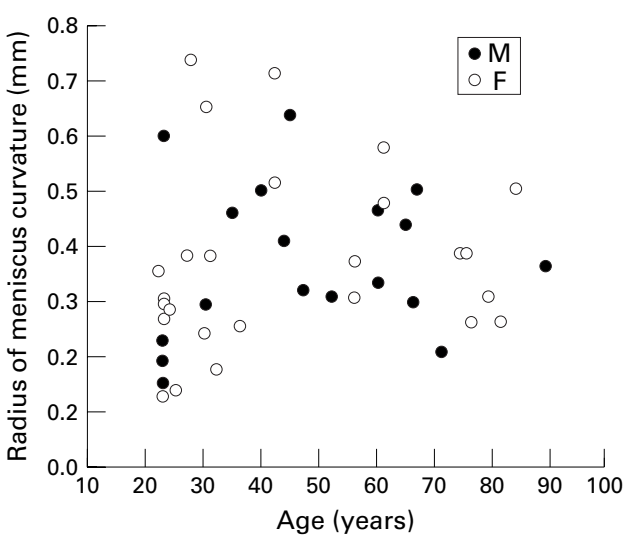

Figure 6 Scatter plot of measured radius of curvature against age of subject.

for photography conformed to a circular profile. Calibration of the video system was achieved by imaging a standard graticule. Only qualitative findings from video meniscometry are reported here.

RADIUS OF CURVATURE OF THE HUMAN TEAR MENISCUS

A representative example of a normal meniscus is shown in Figure 5. Curvature was measured over a small central region, assumed to approximate to a circular profile; six parallel black and white lines, free from distortion, were selected to obtain an average line width. From this, the normal tear meniscus radius was calculated to be mean 0.365 (SD 0.153) $\mathrm{mm}$ (range $0.128-0.736 \mathrm{~mm} ; \mathrm{n}=45$ ) based on the theoretical equation. No significant differences were found in the radius of meniscus curvature with age or sex $\left(R^{2}=0.020\right.$, simple regression analysis; $\mathrm{p}=0.300$, unpaired $t$ test). A scatter plot is shown in Figure 6.

There was no significant difference in curvature between two consecutive photographs of the meniscus taken at an interval of 20 seconds ( $p=0.847$ (paired $t$ test); first photograph: mean 0.365 (SD 0.153) $\mathrm{mm}, \mathrm{n}=45$ ), second photograph: $0.367(0.132) \mathrm{mm}(\mathrm{n}=45))$. 

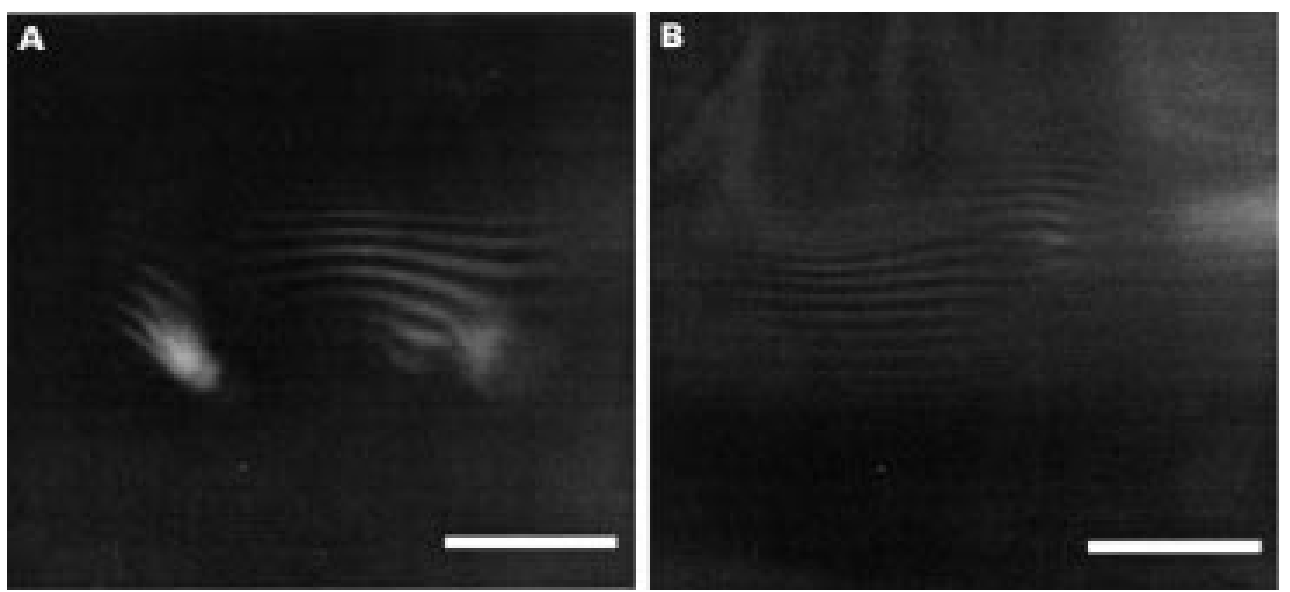

Figure 7 Representative examples of variations in the meniscus shape; (A) forward serrations, $(B)$ doubling; bars $=500$ $\mu m$.

VARIATIONS IN MENISCUS SHAPE

Video imaging allowed changes in meniscus morphology to be studied in more detail and at higher magnification. The meniscus shape varies from subject to subject, and along the length of the lid, but the horizontal lines of the target image were stable at a given location for periods of several minutes, with the patient relaxed and blinking spontaneously. Although the lid tear interface is usually depicted as curvilinear, there were some variations in meniscus form in normal subjects including microirregularities at the mucocutaneous junction. A common feature was the presence of forward serrations which distorted the horizontal stripes of the image. The serrations consisted of forward projections of the tear film across the mucocutaneous junction at the site of the meibomian orifices and do not appear to have been described previously. We suggest that these represent an interaction between the lipid of the preocular tear film and that of the oil pools overlying the orifices. They were detected in 10 out of the 45 cases examined $(22 \%)$. More rarely, multiple reflections were seen, suggesting the presence of discontinuities

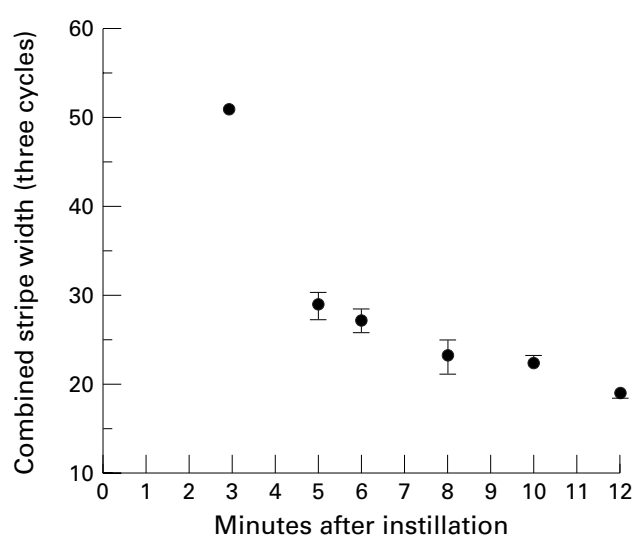

Figure 8 Example showing the change in the tear meniscus curvature, after instillation of a single drop of Celluvisc (1\% carboxymethylcellulose sodium, Allergan). Ordinate: Stripe width measured directly from the TV monitor using a multistripe target. The width of three black and white cycles is shown. Abscissa: Time in minutes. Average width (mm) (SD) (error bars are hidden by the symbols at 3, 10, and 12 minutes). in the meniscus curvature. Representative examples of meniscus shape are shown in Figure 7.

\section{VIDEO MENISCOMETRY}

Video meniscometry, used in six subjects, confirmed the features observed with the photographic technique but also allowed events to be followed in detail over time and to be reviewed. The meniscus shape, with gaze in the primary position, was found to be very stable over periods of 15 minutes or more. Meniscus curvature varied with gaze and was smaller in downgaze than upgaze, confirming our findings using the photographic system, which showed that the radii of the tear meniscus in maximum upgaze, level gaze, and downgaze in one subject were $0.358,0.332$, and $0.192 \mathrm{~mm}$, respectively. Maintenance of eye position is important for the repeatability of the method. The interference pattern of the preocular oil film was visible on video meniscometry, particularly when the meniscus had been expanded by drop instillation. Occasionally, small oil globules were seen to float upwards across the surface of the meniscus as described by Wolff, ${ }^{22}$ and delivery was observed as a burst of oil emanating from a point on the lid margin assumed to overlie a meibomian orifice.

Blinking causes a disturbance in the meniscus, in that meniscus curvature was more concave at the start of the upstroke of the blink but was quickly restored to a less concave configuration which was maintained between blinks.

The effect on meniscus curvature, of instilling small volumes of fluid into the conjunctival sac, was studied in a few subjects. Instillation of a drop of saline or tear substitute $(5-30 \mu \mathrm{l})$ expanded the tear meniscus for a short time. The target image initially disappeared for a period proportional to the volume and viscosity of the drop, presumably due to the creation of a transient, convex meniscus. This was followed by the reappearance of greatly magnified reflections of the target stripes, implying a swollen, shallowly concave meniscus. With time the image size gradually returned to baseline. An example is shown in Figure 8. Detailed 
studies of persistence time studied by reflective meniscometry will be reported elsewhere.

Absence of blinking for 2-4 minutes stimulated reflex tearing and resulted in meniscus swelling, which could be restored to normal with a single blink.

\section{Discussion}

This study has established a novel, noninvasive method to measure tear meniscus curvature, by both photographic and video techniques. In the normal meniscus, the individual stripes of the target imaged in the central, well focused region, are closely similar in width (Fig 5). The images of the peripheral stripes are poorly focused so that measurement is not feasible. The study of Mainstone et al ${ }^{16}$ suggested that the meniscus, captured in slit image, has a parabolic or elliptical cross section, and this may be the case. Our calculation assumes a circular profile in the mid-zone of the meniscus and does not measure curvature over the full height. The good agreement shown between the manual and theoretical methods of measurement used in the present study, confirms that the zone selected lies in a region of the meniscus with near circular cross section.

The radius of curvature of the human tear meniscus was found to be $0.365 \mathrm{~mm}$, in a group of 45 normal subjects. This value is lower than that reported by Mainstone et $a l^{16}$ (mean 0.545 (SD 0.259), $\mathrm{n}=15$ ) and probably reflects the non-invasive nature of the present method, which avoids fluorescein instillation.

Mishima et al ${ }^{1}$ demonstrated that overall tear volume is correlated with tear secretion rate, and it might be expected that meniscus volume would reflect this relation. However, no relation was demonstrated between the radius of meniscus curvature and age in this small study, which is in contrast with the reported fall in aqueous tear secretion ${ }^{123}$ or rise in tear evaporation ${ }^{23}$ which occurs with age. Although the lid margin becomes thicker with age, no age related changes in the morphology of the mucocutaneous junction have been observed. ${ }^{24}$ The influence of pathological change in the mucocutaneous junction remains to be investigated.

Real time changes of meniscus curvature were observed by video meniscometry. The reduction in meniscus radius observed during the upstroke of the blink suggests that, over this time, the upper lid momentarily draws tear fluid into the preocular tear film, which is then restored to the meniscus for the greater part of the interblink period. This is in keeping with the predictions of Wong et al. ${ }^{25}$ It cannot be excluded that fluid is also transferred from the tear sac to the meniscus, after the blink. Between blinks the meniscus curvature was stable, but was influenced by gaze direction. Conditions of measurement must therefore be carefully controlled to obtain consistent readings. Using the photographic system, a single flash did not modify meniscus curvature significantly, which confirmed both the repeatability of the method in the undisturbed subject and its minimally invasive nature with the relatively low flash intensity of the current equipment. It may be that a small reflex increase in tear secretion and volume occurred which was not detected by the system, but in other studies we have been able to detect expansion of the meniscus after deliberate instillation of as little as $1 \mu \mathrm{l}$ of saline. An increase in tear volume by instillation of a single drop of artificial tears $(25 \mu \mathrm{l})$ was readily detected by the system. The rate of recovery of the profile as determined by stripe width, promises to provide a method to follow retention time of tear substitutes of differing viscosity (Fig 8).

In the normal subjects there were some variations in meniscus shape; forward serrations were a common feature, overlying the meibomian orifices. We hypothesise that lipid accumulating at the orifices interacts with the meniscus, destroying its concave profile. It may be that this feature varies along the lid margin with time, according to the state of delivery at a given orifice.

We are currently using reflective meniscometry in a number of ways: (1) to develop the instrument as a tool for the diagnosis of dry eye; (2) to determine recovery time after drop instillations of relevance to the sequencing of dry eye tests (in a recent study, for instance, we found the radius of meniscus curvature to be significantly lower in dry eye patients $(n=32$; $r=0.25$ (SE 0.015)) than in normal subjects $\left.(\mathrm{n}=45 ; r=0.36526(0.023))^{26}\right)$; (3) to compare the relative retention time of tear substitutes; and (4) to detect the adequacy of punctal occlusion in dry eye subjects. In conclusion, meniscometry appears to be a promising new method for investigating the tear film and its disorders, which can provide information about the undisturbed tear meniscus in vivo.

\section{Appendix}

THEORETICAL BACKGROUND FOR OBTAINING THE RADIUS OF MENISCUS CURVATURE

The theoretical optical system is shown Figure 9, where $\mathrm{T}, \mathrm{C}, \mathrm{I}$, and F indicate the target, the centre of the expected circular portion of the meniscus, the image position, and the focus of the convex meniscus mirror, respectively.

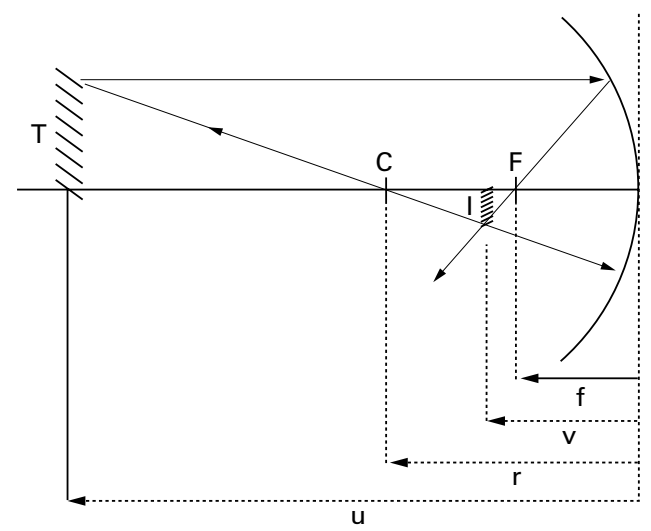

Figure 9 Ray diagram for theoretical determination of the radius of meniscus curvature. $T=$ target; $C=$ centre of the expected circular portion of the meniscus; $I=$ image; $F=$ focal point of the convex meniscus mirror; $u=$ target distance; $v=$ image distance; $r=$ radius of meniscus curvature; $f=$ focal distance. 
According to the convex mirror formula ${ }^{21}$

$$
1 / \mathrm{u}+1 / \mathrm{v}=1 / \mathrm{f}
$$$$
\text { and } \mathrm{f}=\mathrm{r} / 2
$$

where $\mathrm{u}, \mathrm{v}, \mathrm{r}$, and $\mathrm{f}$ are target distance, image distance, radius of meniscus curvature, and focal distance, respectively.

From (A1) and (A2)

$1 / \mathrm{u}+1 / \mathrm{v}=2 / \mathrm{r}$

Using the proportional relation indicated in Figure 9 and (A3), the magnification of the image, $M$, is expressed as:

$$
\mathrm{M}=\mathrm{i} / \mathrm{t}=(\mathrm{r}-\mathrm{v}) /(\mathrm{u}-\mathrm{r})=\mathrm{l}^{\prime} / 1
$$

where the width of a stripe of the target and image are $t$ and $i$, respectively.

From (A3) and (A4), and putting $1 / \mathrm{u}=\mathrm{L}$

$$
r=2 /(\mathrm{L}+\mathrm{L} / \mathrm{M})
$$

We thank Elaine Grande and Paul Parker for their technical assistance in preparing photographs. The authors also wish to thank all the subjects who participated in the study.

1 Mishima S, Gasset A, Klyce SD Jr, et al. Determination of tear volume and tear flow. Invest Ophthalmol 1966;5:264-76. 2 Farris RL, Stuchell RN, Mandel ID. Basal and reflex human ear analysis. I. Physical measurements: osmolarity, basal volumes, and reflex tear rate. Ophthalmology 1981;88:852-7.

3 Zappia IJ, Milder B. Lacrimal drainage function. Am $\mathcal{F} O p h-$ thalmol 1972;74:154-9.

4 Scherz W, Doane MG, Dohlman CH. Tear volume in normal eyes and keratoconjunctivitis sicca. Graefes Arch Clin Exp Ophthalmol 1974;192:141-50.

5 Mishima S, Kubota Z, Farris RL. The tear flow dynamics in normal and keratoconjunctivitis sicca cases. Excerpta Medica Int Cong Ser 1970;222:1801-5.

6 Van Bijsterveld OP. Diagnostic tests in the sicca syndrome. Arch Ophthalmol 1969;82:10-14.

Arch Ophthalmol 1969;82:10-14. symptoms of keratoconjunctivitis sicca. Am $\mathcal{f}$ Ophthalmol 1988;106:570-4.
8 Schirmer O. Studien zur Physiologie und Pathologie der Tränenabsonderung und Tränenabfuhr. Graefes Arch Klin Exp Ophthalmol 1903;56:197-291.

9 Hamano H, Hori M, Hamano T, et al. A new method for measuring tears. CLAO ₹ 1983;9:281-9.

$10 \mathrm{McDonald}$ JE, Brubaker S. Meniscus-induced thinning of tear films. Am f Ophthalmol 1971;72:139-46.

11 Holly FJ, Lemp MA. Tear physiology and dry eyes. Surv Ophthalmol 1977;22:69-87.

12 Lamberts DW, Foster CS, Perry HD. Schirmer test after topical anesthesia and the tear meniscus height in normal eyes. Arch Ophthalmol 1979;97:1082-5.

13 Taylor HR, Louis WJ. Significance of tear function test abnormalities. Ann Ophthalmol 1980;12:531-5.

14 Port MJA, Asaria TS. Assessment of human tear volume. $\mathcal{7}$ Br Contact Lens Assoc 1990;13:76-82.

15 Khurana AK, Chaudhary R, Ahluwalia BK, et al. Tear film profile in dry eye. Acta Ophthalmol (Copenh) 1991;69:7986

16 Mainstone JC, Bruce AS, Golding R. Tear meniscus measurement in the diagnosis of dry eye. Curr Eye Res 1996;15:653-61.

17 Cho P. The cotton thread test: a brief review and a clinical study of its reliability on Hong Kong Chinese. Optom Vis Sci 1993;70:804-8.

18 Loran DFC, French CN, Lam SY, et al. Reliability of the wetting value of tears. Ophthal Physiol Opt 1987;7:53-6.

19 Bron AJ, Tiffany JM, Yokoi N, et al. Meniscometry: a new method to measure tear meniscus curvature. (ARVO Abstracts) Invest Ophthalmol Vis Sci 1997;38(4, Suppl): 151.

20 Brown N. Macrophotography of the anterior segment of the eye. Br f Ophthalmol 1970;54:697-701.

21 Freeman MH. Optics. 10th ed. London: Butterworths, 1990:54-83.

22 Wolff $\mathrm{E}$. The muco-cutaneous junction of the lid-margin and the distribution of the tear fluid. Trans Ophthalmol Soc UK 1946;66:291-308.

23 Mathers WD, Lane JA, Zimmerman MB. Tear film changes associated with normal aging. Cornea 1996;15:229-334.

24 Hykin PG, Bron AJ. Age-related morphological changes in lid margin and meibomian gland anatomy. Cornea 1992;11: 334-42.

25 Wong H, Fatt I, Radke CJ. Deposition and thinning of the human tear film. 7 Colloid Interface Sci 1996;184:44-51.

26 Bron AJ, Yokoi N, Tiffany JM, et al. . The clinical value of reflective meniscometry in dry eye diagnosis. Invest Ophthalmol Vis Sci 1998;39:65 (Abstract 292). 